\title{
Sequential Cycloaddition Approach to the Tricyclic Core of Vibsanin E. Total Synthesis of 5-epi-10-epi- Vibsanin E
}

\author{
Huw M. L. Davies*, Øystein Loe and Douglas G. Stafford \\ Department of Chemistry, University at Buffalo, The state University of New York, Buffalo, NY 14260-300
}

\section{Supporting Information}

Section A: General Information

S-2

Section B: Experimental Procedures

S-3

Section C: Relative Stereochemistry of 5-epi-10-epi-Vibsanin E

S-16

Section D: ${ }^{1} \mathrm{H}$ NMR Spectra

S-18 


\section{Section A: General Information.}

Commercial reagents from Aldrich Chemical Company and Acros Organics were purchased at the highest commercial purity and used without further purification unless otherwise noted. All moisture sensitive reactions were performed using glassware flame dried under vacuum and purged with argon prior to use. Tetahydrofuran, hexanes and dichloromethane were used directly from the solvent purification system, where solvent was passed through two columns of activated alumina, purchased from MBRAUN. All other solvents were of reagent grade. Solvents used in rhodium carbenoid transformations were degassed with argon for 10-15 min. prior to use. Organic reaction mixtures were concentrated on a Buchi rotary evaporator. Analytical thin layer chromatography was performed on $250 \_\mathrm{m}$ Whatman silica gel (Aluminum backing, UV $254 \mathrm{~nm}$ ) plates using UV light or $50 \%$ sulfuric acid in methanol as visualizing agents. Column chromatography was carried out using E. Merck silica 60 (230 - 400 mesh) or ICN 60 (32 - 64 mesh) following the method of Still. ${ }^{1}$

${ }^{1}$ H NMR spectra were recorded on a Varian Nuclear Magnetic Resonance spectrometer at 400 or $500 \mathrm{MHz}$ and ${ }^{13} \mathrm{C}$ NMR spectra were recorded at 75 or $125 \mathrm{MHz}$, with the sample solvent being $\mathrm{CDCl}_{3}$ or $\mathrm{C}_{6} \mathrm{D}_{6}$. The $\mathrm{CDCl}_{3}$ samples were calibrated at the solvent peak at $7.27 \mathrm{ppm}$ for the ${ }^{1} \mathrm{H}$ NMR spectra, for the ${ }^{13} \mathrm{C}$ NMR spectra the middle solvent peak was set at $77 \mathrm{ppm}$. The $\mathrm{C}_{6} \mathrm{D}_{6}$ samples were calibrated at $0 \mathrm{ppm}$ with TMS as internal standard or at the solvent peak at $7.157 \mathrm{ppm}$ for the ${ }^{1} \mathrm{H}$ NMR spectra. The ${ }^{13} \mathrm{C}$ NMR spectra were set likewise at $0 \mathrm{ppm}$ with TMS or at $128.026 \mathrm{ppm}$ for the middle peak. The following abbreviations are used to explain the multiplicities: s, singlet; d, doublet; t, triplet; q,

${ }^{1}$ Still, W. C.; Kahn, M.; Mitra, A. J. J. Org. Chem. 1978, 43, 2923 
quartet; dd, doublet of doublets; dt, doublet of triplets; ddd, doublet of doublet of doublets. Infrared spectra were recorded on a Nicolet Impact 420 FT-IR spectrometer. High resolution mass spectra were obtained from the Mass Spectroscopy Facility at the University at Buffalo, The State University of New York.

\section{Section B: Experimental Procedures:}

\section{Synthesis of 4-Methyl-4-(4-methyl-pent-3-enyl)-cyclohepta-1,5-dienecarboxylic acid methyl ester (5)}

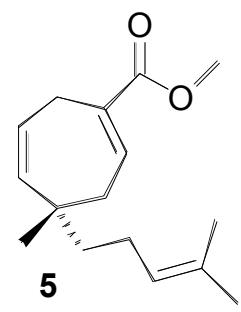

The catalyst $\mathrm{Rh}_{2}(\mathrm{OOct})_{2}(741 \mathrm{mg}, 0.952 \mathrm{mmol}, 0.5 \mathrm{~mol} \%)$ was dissolved in the triene 3 (157 g, $1.05 \mathrm{~mol}$ ) (due to much better solubility than in hexane). This mixture was diluted with hexane $(1.90 \mathrm{~L})$ to furnish a homogenous dark green solution that was degassed by bubbling argon through $(20 \mathrm{~min}$.). The diazo compound 4 (24.0 g, $0.190 \mathrm{~mol})$ was dissolved in cold $\left(-20^{\circ} \mathrm{C}\right)$ hexane $(0.30 \mathrm{~L})$ and added dropwise to the alkene/ rhodium solution from an acetone/ dry-ice cooled addition funnel. After the addition was complete (1 h) the reaction was left stirring overnight at room temperature. The organics were concentrated under reduced pressure and the catalyst was removed by filtration through a short silica plug ( $10 \%$ diethyl ether in hexane). The residue was dissolved in toluene (150 $\mathrm{mL}$ ) and heated at reflux $(5 \mathrm{~h})$ in order to force the Cope rearrangement to completion. 
The toluene was removed under reduced pressure and the product was purified by silica gel chromatography ( $5 \%$ diethyl ether in hexane). This furnished the cycloheptadiene 5 in $62 \%$ yield $(29.2 \mathrm{~g}, 0.118 \mathrm{~mol})$.

Alternatively,

To a stirred solution of the triene $3(906 \mathrm{mg}, 6.0 \mathrm{mmol})$, and $\mathrm{Rh}_{2}(R-\mathrm{DOSP})_{4}(22 \mathrm{mg}$, $0.012 \mathrm{mmol})$ in hexane $(30 \mathrm{ml})$ at $23{ }^{\circ} \mathrm{C}$ under argon, vinyldiazoacetate $4(148 \mathrm{mg}, 1.17$ mmol) in hexane $(25 \mathrm{ml})$ at $0{ }^{\circ} \mathrm{C}$ was added via cannula over $20 \mathrm{~min}$. After $40 \mathrm{~h}$. the solvent was removed under reduced pressure. Purification by silica gel chromatography (pentane/diethyl ether 19:1) gave $5(202 \mathrm{mg}, 0.811 \mathrm{mmol})$ in $69 \%$ yield and $64 \%$ ee (determined by GC:_-TA; $100{ }^{\circ} \mathrm{C}, 21.4 \mathrm{psi}, 2.1 \mathrm{~mL} / \mathrm{min} ; \mathrm{T}_{\mathrm{R}}=185.4 \mathrm{~min}$ (minor), 189.7 $\min$ (major). []$^{25}{ }_{\mathrm{D}}=-11^{\circ}\left(\mathrm{c} 2.16, \mathrm{CHCl}_{3}\right){ }^{1} \mathrm{H} \mathrm{NMR}\left(\mathrm{CDCl}_{3}, 400 \mathrm{MHz}, \delta\right) 7.08(\mathrm{dd}, 1 \mathrm{H}, J$ $=7.4,7.4 \mathrm{~Hz}), 5.50(\mathrm{dt}, 1 \mathrm{H}, J=11.6,5.2 \mathrm{~Hz}), 5.29(\mathrm{~d}, 1 \mathrm{H}, J=11.6 \mathrm{~Hz}), 5.06(\mathrm{t}, 1 \mathrm{H}, J=$ $6.6 \mathrm{~Hz}) 3.71(\mathrm{~s}, 3 \mathrm{H}), 3.11(\mathrm{~d}, 2 \mathrm{H}, J=5.2 \mathrm{~Hz}), 2.50(\mathrm{dd}, 1 \mathrm{H}, J=13.2,7.4 \mathrm{~Hz}), 2.24$ (dd, $1 \mathrm{H}, J=13.2,7.4 \mathrm{~Hz}), 1.95$ (ddd, 2H, $J=11.2,6.6,6.4 \mathrm{~Hz}), 1.65(\mathrm{~s}, 3 \mathrm{H}), 1.58(\mathrm{~s}, 3 \mathrm{H})$, $1.30(\mathrm{dd}, 2 \mathrm{H}, J=11.2,6.4 \mathrm{~Hz}), 0.98$ (s, 3H). ${ }^{13} \mathrm{C}$ NMR $\left(\mathrm{CDCl}_{3}, 75 \mathrm{MHz}, \mathrm{DEPT}, \delta\right) 167.5$ $\left(4^{\circ}\right), 141.7\left(3^{\circ}\right), 139.6\left(3^{\circ}\right), 135.2\left(4^{\circ}\right), 131.3\left(3^{\circ}\right), 124.6\left(3^{\circ}\right), 123.2\left(3^{\circ}\right), 51.7\left(1^{\circ}\right), 43.2$ $\left(2^{\circ}\right), 37.7\left(4^{\circ}\right), 37.2\left(2^{\circ}\right), 27.9\left(1^{\circ}\right), 25.7\left(2^{\circ}\right), 25.6\left(1^{\circ}\right), 23.1\left(2^{\circ}\right), 17.5\left(1^{\circ}\right)$. IR (neat) 3009, 2956, 2924, 2860, 1715, $1651 \mathrm{~cm}^{-1}$. MS $\mathrm{m} / z$ (relative intensity) 248 (3), 233 (6), 205 (12), 173 (16), 145 (25), 133 (27), 119 (46), 105 (100), 91 (78). Anal. Calcd. for $\mathrm{C}_{16} \mathrm{H}_{24} \mathrm{O}_{2}$ : C, 77.38; H, 9.74. Found : C, 77.17; H, 9.66 


\section{Synthesis of 4-Methyl-4-(4-methyl-pent-3-enyl)-cyclohepta-1,5-dienecarbaldehyde}

(6)

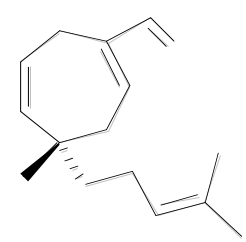

The ester 5 (19.0 g, $76.6 \mathrm{mmol})$ was dissolved in THF $(500 \mathrm{~mL})$ and DIBAL-H $(200 \mathrm{~mL}$, 1.0 M, $0.20 \mathrm{~mol})$ was added dropwise from an addition funnel $\left(30 \mathrm{~min}\right.$.) at $0{ }^{\circ} \mathrm{C}$. The reaction was stirred over night and allowed to warm to room temperature. The crude mixture was quenched with a water/ ice solution $(500 \mathrm{~mL})$ and diluted with $\mathrm{HCl}(100$ $\mathrm{mL}, 10 \%)$. The aqueous phase was extracted with diethyl ether ( 3 X $150 \mathrm{~mL})$. The organics were back washed with water $(200 \mathrm{~mL})$ and brine $(200 \mathrm{~mL})$ before dried over magnesium sulfate and the solvent was evaporated. This gave the crude alcohol (17 g) that was used in the Swern oxidation without further purification. ${ }^{1} \mathrm{H}$ NMR $\left(\mathrm{CDCl}_{3}, 500\right.$ $\mathrm{MHz}, \delta) 5.77(\mathrm{t}, 1 \mathrm{H}, J=7.0 \mathrm{~Hz}), 5.51(\mathrm{dt}, 1 \mathrm{H}, J=11.5,6.0 \mathrm{~Hz}), 5.30(\mathrm{~d}, 1 \mathrm{H}, J=11.5$ $\mathrm{Hz}), 5.10(\mathrm{t}, 1 \mathrm{H}, J=6.5 \mathrm{~Hz}), 4.03(\mathrm{~s}, 2 \mathrm{H}), 2.85(\mathrm{dd}, 1 \mathrm{H}, J=5.0,18.5 \mathrm{~Hz}), 2.75(\mathrm{dd}, 1 \mathrm{H}, J$ $=5.0,18.5 \mathrm{~Hz}), 2.38(\mathrm{dd}, 1 \mathrm{H}, J=6.5,16.5 \mathrm{~Hz}), 2.11(\mathrm{dd}, 1 \mathrm{H}, J=6.5,16.5 \mathrm{~Hz}), 2.00-$ $1.95(\mathrm{~m}, 2 \mathrm{H}), 1.69(\mathrm{~s}, 3 \mathrm{H}), 1.61(\mathrm{~s}, 3 \mathrm{H}), 1.49(\mathrm{~s}$, broad, $1 \mathrm{H}), 1.32(\mathrm{~m}, 2 \mathrm{H}), 0.99(\mathrm{~s}, 3 \mathrm{H})$.

Oxalyl chloride (13 mL, $18.9 \mathrm{~g}, 0.149 \mathrm{~mol})$ was dissolved in dichloromethane (500 mL) and cooled to $-78{ }^{\circ} \mathrm{C}$. Then DMSO $(17 \mathrm{~mL}, 18.7 \mathrm{~g}, 0.24 \mathrm{~mol})$ was added and the solution was stirred for $15 \mathrm{~min}$ at $-78{ }^{\circ} \mathrm{C}$. The alcohol $(17 \mathrm{~g}, 77 \mathrm{mmol})$ dissolved in dichloromethane $(200 \mathrm{~mL})$ was added dropwise at $-78{ }^{\circ} \mathrm{C}$ and then stirred for $30 \mathrm{~min}$. Triethylamine $(120 \mathrm{~mL})$ was added over $10 \mathrm{~min}$ and then the reaction was stirred for 5 
min at $-78{ }^{\circ} \mathrm{C}$. The mixture was allowed to warm to room temperature and the reaction mixture was extracted with water $(100 \mathrm{~mL})$ and the organics were dried over sodium sulfate. After removal of the solvent and purification by silica gel chromatography $(5 \%$ ethyl ether in hexane) the aldehyde $6(15.1 \mathrm{~g}, 69.3 \mathrm{mmol})$ was obtained in $90 \%$ yield. ${ }^{1} \mathrm{H}$ NMR $\left(\mathrm{CDCl}_{3}, 500 \mathrm{MHz}, \delta\right) 9.39(\mathrm{~s}, 1 \mathrm{H}), 6.84(\mathrm{t}, 1 \mathrm{H}, J=6.7 \mathrm{~Hz}), 5.52(\mathrm{dt}, 1 \mathrm{H}, J=11.6$, $17.1 \mathrm{~Hz}), 5.38(\mathrm{~d}, 1 \mathrm{H}, J=11.6 \mathrm{~Hz}), 5.09(\mathrm{t}, 1 \mathrm{H}, J=7.0 \mathrm{~Hz}), 3.08(\mathrm{dd}, 1 \mathrm{H}, J=5.2,19.5$ $\mathrm{Hz}), 3.03(\mathrm{dd}, 1 \mathrm{H}, J=5.2,19.5 \mathrm{~Hz}), 2.68(\mathrm{dd}, 1 \mathrm{H}, J=6.7,13.7 \mathrm{~Hz}), 2.45(\mathrm{dd}, 1 \mathrm{H}, J=$ 6.7, $13.7 \mathrm{~Hz}), 2.02-1.97(\mathrm{~m}, 2 \mathrm{H}), 1.69(\mathrm{~s}, 3 \mathrm{H}), 1.61(\mathrm{~s}, 3 \mathrm{H}), 1.40-1.33(\mathrm{~m}, 2 \mathrm{H}), 1.05(\mathrm{~s}$, 3H). ${ }^{13} \mathrm{C} \mathrm{NMR}\left(\mathrm{CDCl}_{3}, 125 \mathrm{MHz}, \delta\right)$ 193.0, 153.3, 145.4, 139.4, 131.5, 124.4, 122.8, 43.2, 38.2 (2C), 28.0, 25.6, 23.1, 23.0, 17.5. IR (neat, $\mathrm{cm}^{-1}$ ) 3350 (overtone, Fermi), 3004, 2962, 2919, 2860, 2722, 1683, 1651, 1454, 1380, 1162, 725. MS (EI) $m / z$ (relative intensity) 218 (45), 175 (54), 107 (100), 105 (69), 91 (75), 79 (55), HRMS calcd. for $\mathrm{C}_{15} \mathrm{H}_{22} \mathrm{O} 218.1665$, found 218.1676.

\section{Synthesis of 1,5,5-Trimethyl-6-oxa-tricyclo[6.3.2.0 $\left.{ }^{4,13}\right]$ trideca-7,10-diene (7)}

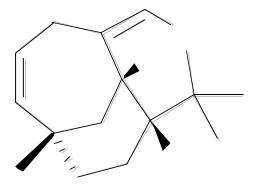

The aldehyde 6 (15.1 g, $69.3 \mathrm{mmol})$ was dissolved in dichloromethane $(500 \mathrm{~mL})$ and cooled to $-78{ }^{\circ} \mathrm{C}$ and $\mathrm{BF}_{3} \cdot \mathrm{OEt}_{2}(30 \mathrm{~mL}, 0.24 \mathrm{~mol})$ was added. The reaction was stirred at $-78^{\circ} \mathrm{C}$ for $20 \mathrm{~min}$. and quenched by addition of water $(500 \mathrm{~mL})$. The reaction mixture was extracted with dichloromethane ( 3 X $250 \mathrm{~mL})$ and the organics were dried over 
magnesium sulfate. The crude product was purified on a silica gel column (4\% diethyl ether in hexane) to afford the tricyclic system 7 in $84 \%$ yield $(13.0 \mathrm{~g}, 59.6 \mathrm{mmol}) .{ }^{1} \mathrm{H}$ NMR $\left(\mathrm{CDCl}_{3}, 500 \mathrm{MHz}, \delta\right) 6.03(\mathrm{~d}, 1 \mathrm{H}, J=2.1 \mathrm{~Hz}), 5.74(\mathrm{ddd}, 1 \mathrm{H}, J=1.8,11.1,11.1$ Hz), $4.99(\mathrm{~d}, 1 \mathrm{H}, J=11.1 \mathrm{~Hz}), 2.97(\mathrm{~d}, 1 \mathrm{H}, J=15.6 \mathrm{~Hz}), 2.79$ (s, broad, 1H), 2.32 (m, 2H), $1.55-1.47(\mathrm{~m}, 2 \mathrm{H}), 1.39(\mathrm{dd}, 1 \mathrm{H}, J=4.0,12.8 \mathrm{~Hz}), 1.31-1.21(\mathrm{~m}, 2 \mathrm{H}), 1.25(\mathrm{~s}$, $3 \mathrm{H}), 1.24(\mathrm{~s}, 3 \mathrm{H}), 1.14(\mathrm{dt}, 1 \mathrm{H}, J=4.0,12.8 \mathrm{~Hz}), 0.96(\mathrm{~s}, 3 \mathrm{H}){ }^{13} \mathrm{C} \mathrm{NMR}\left(\mathrm{CDCl}_{3}, 125\right.$ MHz, $\delta$ (multiplicity)) 137.6 (d), 136.7 (d), 129.1 (d), 112.0 (s), 76.1 (s), 41.0 (d), 38.1 (2C) (t,t), $37.1(\mathrm{~s}), 33.1(\mathrm{q}), 31.2(\mathrm{~d}), 28.3(\mathrm{t}), 27.7(\mathrm{q}), 26.0(\mathrm{q}), 19.8(\mathrm{t})$. IR (neat, $\left.\mathrm{cm}^{-1}\right)$ $3053,2928,2866,1660,1453,1370,1230,1147,940,805$. MS (EI) $\mathrm{m} / z$ (relative intensity) 218 (100), 136 (67), 107 (59), 105 (65), 91 (72), 69 (53). HRMS calcd. for $\mathrm{C}_{15} \mathrm{H}_{22} \mathrm{O} 218.1665$, found 218.1663.

\section{Synthesis of 1,5,5-Trimethyl-6-oxa-tricyclo[6.3.2.0 $\left.{ }^{4,13}\right]$ tridec-10-ene (8)}

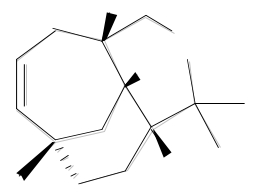

To a stirred solution of the diene $7(13.0 \mathrm{~g}, 59.6 \mathrm{mmol})$ in acetic acid $(500 \mathrm{~mL})$ at room temperature was added $\mathrm{NaCNBH}_{3}(17 \mathrm{~g}, 270 \mathrm{mmols})$ and the solution was stirred for 20 min. The same quantity of $\mathrm{NaCNBH}_{3}(17 \mathrm{~g}, 270 \mathrm{mmol})$ was added again and stirred for 20 minutes. At this point the reaction was quenched with water $(1 \mathrm{~L})$ and extracted with hexane $(2 \times 250 \mathrm{~mL})$. The organics were dried over magnesium sulfate and evaporated to yield the crude product. The mixture was purified on a silica column with $5 \%$ diethyl ether in hexane to yield the desired compound 8 in $80 \%$ yield $(11.0 \mathrm{~g}, 50.0 \mathrm{mmol}) .{ }^{1} \mathrm{H}$ 
NMR $\left(\mathrm{CDCl}_{3}, 400 \mathrm{MHz}, \delta\right) 5.54(\mathrm{ddd}, 1 \mathrm{H}, \mathrm{J}=2.4,7.3,12.2 \mathrm{~Hz}), 5.42(\mathrm{ddd}, 1 \mathrm{H}, \mathrm{J}=1.5$, 2.7, $12.2 \mathrm{~Hz}$ ), 3.83 (dd, 1H, J = 2.6, 10.6 Hz), 3.35 (d, 1H, J = 10.6 Hz), 2.85 (ddd, 1H, J $=3.3,7.0,14.4 \mathrm{~Hz}), 2.39(\mathrm{~d}, 1 \mathrm{H}$, broad, $\mathrm{J}=4.4 \mathrm{~Hz}), 2.23(\mathrm{ddd}, 1 \mathrm{H}, \mathrm{J}=7.0,11.7,14.4)$, $1.77-1.68(\mathrm{~m}, 3 \mathrm{H}), 1.57-1.47(\mathrm{~m}, 3 \mathrm{H}), 1.31(\mathrm{~s}, 3 \mathrm{H}), 1.30-1.21(\mathrm{~m}, 2 \mathrm{H}), 1.20(\mathrm{~s}, 3 \mathrm{H})$, $1.02(\mathrm{~s}, 3 \mathrm{H}) .{ }^{13} \mathrm{C} \mathrm{NMR}\left(\mathrm{CDCl}_{3}, 125 \mathrm{MHz}, \delta\right)$ 140.1, 127.7, 74.1, 67.6, 43.6, 43.3, 41.3, 35.9, 35.4, 34.2, 33.4, 33.1, 27.7, 24.6, 23.2. IR (neat, $\mathrm{cm}^{-1}$ ) 2946, 2892, 1662, 1454, 1364, 1087. HRMS calcd. for $\mathrm{C}_{15} \mathrm{H}_{24} \mathrm{O} 220.1822$, found 220.1824 .

\section{Synthesis of 1,5,5-Trimethyl-6-oxa-tricyclo[6.3.2.0 $\left.{ }^{4,13}\right]$ tridec-10-en-9-one (9)}

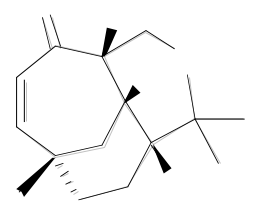

To a stirred solution of the alkene $8(11.0 \mathrm{~g}, 50.0 \mathrm{mmol})$ in 1,4-dioxane $(450 \mathrm{~mL})$ was added $\mathrm{SeO}_{2}(6.66 \mathrm{~g}, 60 \mathrm{mmol})$. The reaction was stirred at reflux $(2 \mathrm{~h})$ and then allowed to cool to room temperature. The reaction was added water $(1 \mathrm{~L})$ and extracted with ether ( 2 X $200 \mathrm{~mL}$ ). The organics were dried over $\mathrm{MgSO}_{4}$ and the solvent was evaporated. The crude product was dissolved in dichloromethane $(500 \mathrm{~mL})$ and $4 \AA$ molecular sieves were added with pyridinium chlorochromate $(25 \mathrm{~g}, 0.12 \mathrm{~mol})$. The reaction was stirred at room temperature $(2 \mathrm{~h})$ before the mixture was concentrated down and diluted with diethyl ether (1L), The mixture was extracted with water (2 X $200 \mathrm{~mL})$ and brine ( 2 X $200 \mathrm{~mL})$. The organics were dried over magnesium sulfate. The crude product was purified on a silica column with 10\% acetone in hexane to yield the ketone 9 in $72 \%(8.5 \mathrm{~g}, 36 \mathrm{mmol})$ yield. ${ }^{1} \mathrm{H}$ NMR $\left(\mathrm{CDCl}_{3}, 500 \mathrm{MHz}, \delta\right) 6.07$ (s, 2H), 4.57 (d, $\left.1 \mathrm{H}, J=11.7 \mathrm{~Hz}\right), 3.60$, (dd, 
$1 \mathrm{H}, J=3.1,11.7 \mathrm{~Hz}), 2.62(\mathrm{~d}, 1 \mathrm{H}, J=4.4 \mathrm{~Hz}), 2.16(\mathrm{t}, 1 \mathrm{H}, J=4.4 \mathrm{~Hz}), 2.08(\mathrm{~d}, 1 \mathrm{H}, J=$ 13.7 Hz), $1.73(\mathrm{dd}, 1 \mathrm{H}, J=5.8,13.7 \mathrm{~Hz}), 1.56(\mathrm{~m}, 2 \mathrm{H}), 1.30(\mathrm{~s}, 3 \mathrm{H}), 1.27(\mathrm{~m}, 2 \mathrm{H}), 1.17$ (s, 3H), 1.15 (m, 1H), $1.12(\mathrm{~s}, 3 \mathrm{H}) .{ }^{13} \mathrm{C} \mathrm{NMR}\left(\mathrm{CDCl}_{3}, 125 \mathrm{MHz}, \delta\right)$ 203.0, 151.9, 131.9, 73.6, 61.4, 50.4, 43.8, 42.9, 38.0, 36.9, 32.2, 31.9, 28.0, 24.1, 19.7. IR (neat, $\mathrm{cm}^{-1}$ ) 2895, 1647, 1455, 1088. HRMS calcd. for $\mathrm{C}_{15} \mathrm{H}_{22} \mathrm{O}_{2} 234.1614$, found 234.1623 .

\section{Synthesis of 1,5,5,11-Tetramethyl-6-oxa-tricyclo[6.3.2.0 $\left.0^{4,13}\right]$ tridecan-9-one (10)}

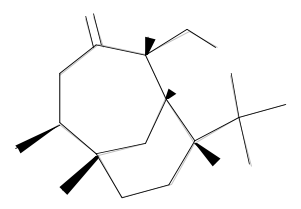

To a stirred solution of activated $\mathrm{CuI}$ (activated under vacuum at $130{ }^{\circ} \mathrm{C}$ for $30 \mathrm{~min}$.) $(0.24 \mathrm{~g}, 1.3 \mathrm{mmol})$ in diethyl ether $(5 \mathrm{~mL})$ at $0{ }^{\circ} \mathrm{C}$ was added methyl lithium $(1.9 \mathrm{~mL}, 1.4$ M, $2.6 \mathrm{mmol}$ ). This was stirred for $20 \mathrm{~min}$. at $0{ }^{\circ} \mathrm{C}$ and a gray homogenous solution resulted. To this solution the ketone $9(91.8 \mathrm{mg}, 0.392 \mathrm{mmol})$ in diethyl ether $(1.5 \mathrm{~mL})$ was added dropwise over $2 \mathrm{~min}$. The reaction was stirred at $0{ }^{\circ} \mathrm{C}$ and allowed to warm slowly to room temperature and stirred overnight before quenched and extracted with a 1:10 solution of $\mathrm{NH}_{4} \mathrm{OH} / \mathrm{NH}_{4} \mathrm{Cl}(2 \mathrm{X} 25 \mathrm{~mL})$, followed by brine $(25 \mathrm{~mL})$. The organics were dried over magnesium sulfate and the crude product was purified on a silica column with $10 \%$ acetone in hexane to yield the desired ketone 10 (74.1 $\mathrm{mg}, 0.296 \mathrm{mmol})$ in $76 \%$ yield. ${ }^{1} \mathrm{H}$ NMR $\left(\mathrm{CDCl}_{3}, 500 \mathrm{MHz}, \delta\right) 4.28(\mathrm{~d}, 1 \mathrm{H}, J=11.9 \mathrm{~Hz}), 3.59(\mathrm{dd}, 1 \mathrm{H}, J=3.4$, $11.9 \mathrm{~Hz}), 2.62(\mathrm{~m}, 2 \mathrm{H}), 2.48(\mathrm{dd}, 1 \mathrm{H}, J=3.4,12.8 \mathrm{~Hz}), 2.20(\mathrm{~m}, 2 \mathrm{H}), 1.72(\mathrm{~m}, 1 \mathrm{H}), 1.60$ (m, 1H), $1.50(\mathrm{~m}, 1 \mathrm{H}), 1.45(\mathrm{dd}, 1 \mathrm{H}, J=5.5,14.6 \mathrm{~Hz}), 1.29(\mathrm{~m}, 1 \mathrm{H}), 1.28(\mathrm{~s}, 3 \mathrm{H}), 1.23$ (m, 1H), 1.18 (m, 1H), 1.13 (s, 3H), 0.99 (d, 3H, $J=7.3 \mathrm{~Hz}), 0.95$ (s, 3H). ${ }^{13} \mathrm{C}$ NMR 
$\left(\mathrm{CDCl}_{3}, 125 \mathrm{MHz}, \delta\right) 213.2,73.3,60.1,50.6,50.1,43.1,41.8,40.5,37.5,33.0,30.6$,

30.2, 27.6, 23.5, 20.2, 19.9. IR (neat, $\mathrm{cm}^{-1}$ ) 2959, 2895, 1698, 1450, 1111. HRMS calcd. for $\mathrm{C}_{16} \mathrm{H}_{26} \mathrm{O}_{2} 250.1927$ found 250.1928

Synthesis of 1,5,12,12-Tetramethyl-11-oxa-tetracyclo $\left[7,6,2,0^{2,7}, 0^{13,17}\right]$ heptadec-4-en8-one (13) and 1,4,12,12-Tetramethyl-11-oxa-tetracyclo[ $\left[7,6,2,0^{2,7}, 0^{13,17}\right]$ heptadec-4en-8-one (14)

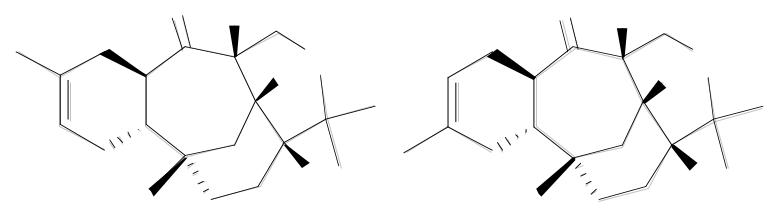

The conjugated ketone 9 (2.21 g, $9.44 \mathrm{mmol})$ was dissolved in isoprene $(200 \mathrm{~mL})$ and exposed to UV light for $15 \mathrm{~h}$ at room temperature. The excess isoprene was removed under reduced pressure and the crude product was purified on a silica gel column with 1 : $30: 69$ ratio of isopropanol: diethyl ether: hexane as eluent. The product was isolated in $91 \%(2.60 \mathrm{~g}, 8.61 \mathrm{mmol})$ yield as a mixture of regioisomers favoring $\mathbf{1 3}$ in a 2:1 ratio.

Spectroscopic data for major product, alkene 13: ${ }^{1} \mathrm{H} \mathrm{NMR}\left(500 \mathrm{MHz}, \mathrm{CDCl}_{3}\right) \delta 5.36(\mathrm{~s}$, broad, $1 \mathrm{H}$ ), 4.10 (dd, 1H, $J=2.4,12.0 \mathrm{~Hz}$ ), 3.73 (dd, 1H, $J=4.6,12.0 \mathrm{~Hz}$ ), 2.93 (ddd, $1 \mathrm{H}, J=5.2,11.6,17.7 \mathrm{~Hz}), 2.57$ (m, broad, 1H), $2.50(\mathrm{~m}, 1 \mathrm{H}), 2.22(\mathrm{~m}$, broad, $1 \mathrm{H}), 2.15$ - 2.12 (m, broad, 2H), $1.98(\mathrm{~m}, 1 \mathrm{H}), 1.93(\mathrm{ddd}, 1 \mathrm{H}, J=2.7,5.8,14.6 \mathrm{~Hz}), 1.82(\mathrm{ddd}, 1 \mathrm{H}$, $J=2.4$ (two), $14.3 \mathrm{~Hz}), 1.71(\mathrm{dd}, 1 \mathrm{H}, J=5.5,14.3 \mathrm{~Hz}), 1.66$ (m, broad, $1 \mathrm{H}), 1.64(\mathrm{~s}, 3 \mathrm{H})$, $1.56(\mathrm{~m}$, broad, $1 \mathrm{H}), 1.46(\mathrm{ddd}, 1 \mathrm{H}, J=4.6,11.6,16.2 \mathrm{~Hz}), 1.38-1.31(\mathrm{~m}, 2 \mathrm{H}), 1.30(\mathrm{~s}$, 
3H), $1.13(\mathrm{~s}, 3 \mathrm{H}), 1.05(\mathrm{~m}, 1 \mathrm{H}), 0.91(\mathrm{~s}, 3 \mathrm{H}) .{ }^{13} \mathrm{C} \mathrm{NMR}\left(125 \mathrm{MHz}, \mathrm{CDCl}_{3}\right) \delta$ 214.8, $132.8,120.3,73.6,61.3,51.6,50.6,46.9,46.0,42.0,37.2,34.0,33.1,32.9,29.7,28.7$, 27.1, 24.3, 22.6, 22.2. IR (neat, $\mathrm{cm}^{-1}$ ) 2906, 1680, 1455, 1367, 1090. HRMS (EI) calcd. for $\mathrm{C}_{20} \mathrm{H}_{30} \mathrm{O}_{2} 302.2240$, found 302.2242 .

Spectroscopic data for minor product, alkene 14: ${ }^{1} \mathrm{H} \mathrm{NMR}\left(500 \mathrm{MHz}, \mathrm{CDCl}_{3}\right) \delta 5.37$ (s, broad, $1 \mathrm{H}), 4.10(\mathrm{dd}, 1 \mathrm{H}, J=2.1,11.9 \mathrm{~Hz}), 3.71(\mathrm{dd}, 1 \mathrm{H}, J=4.3,11.9 \mathrm{~Hz}), 2.82$ (ddd, 1H, $J=5.2,11.0,17.1 \mathrm{~Hz}), 2.56(\mathrm{~s}$, broad, $1 \mathrm{H}), 2.48(\mathrm{~m}, 1 \mathrm{H}), 2.22(\mathrm{~m}, 2 \mathrm{H}), 1.95(\mathrm{~m}$, 3H), $1.83(\mathrm{~d}$, broad, $1 \mathrm{H}, J=14.4 \mathrm{~Hz}), 1.72(\mathrm{dd}, 1 \mathrm{H}, J=5.5,14.4 \mathrm{~Hz}), 1.66$ (s, broad, 3H), $1.55(\mathrm{~m}, 2 \mathrm{H}), 1.35(\mathrm{~m}, 2 \mathrm{H}), 1.29(\mathrm{~s}, 3 \mathrm{H}), 1.13(\mathrm{~s}, 3 \mathrm{H}), 1.06(\mathrm{~m}, 1 \mathrm{H}), 0.93(\mathrm{~s}, 3 \mathrm{H})$. ${ }^{13} \mathrm{C}$ NMR $\left(125 \mathrm{MHz}, \mathrm{CDCl}_{3}\right) \delta$ 214.8, 133.2, 119.8, 73.5, 61.3, 51.4, 50.6, 47.0, 46.2, 42.2, 34.0, 33.6, 33.0, 32.9, 32.8, 29.6, 27.1, 24.3, 23.2, 22.2. IR (neat, $\mathrm{cm}^{-1}$ ) 2906, 1679, 1452, 1367, 1090. HRMS (EI) calcd. for $\mathrm{C}_{20} \mathrm{H}_{30} \mathrm{O}_{2} 302.2240$, found 302.2244 .

Synthesis of 4,5-dihydroxy-1,5,12,12-Tetramethyl-11-oxatetracyclo $\left[7,6,2,0^{2,7}, 0^{13,17}\right]$ heptadec-8-one (15) and 4,5-dihydroxy-1,4,12,12Tetramethyl-11-oxa-tetracyclo[ $\left[7,6,2,0^{2,7}, 0^{13,17}\right]$ heptadec-8-one (16)
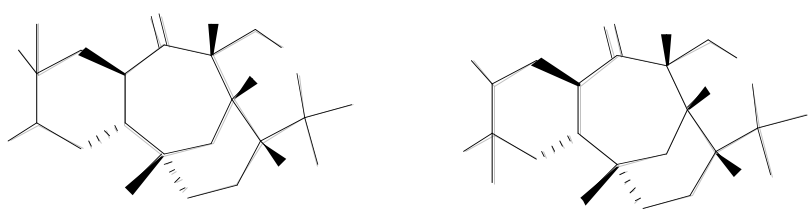

A mixture of the two alkene regioisomers 13 and $\mathbf{1 4}(125 \mathrm{mg}, 0.414 \mathrm{mmol})$ was dissolved in acetone $(4 \mathrm{~mL})$ and water $(0.5 \mathrm{~mL})$ followed by addition of NMO (134 mg, 1.18 mmol) and $\mathrm{OsO}_{4}(0.67 \mathrm{~mL}, 2.5 \mathrm{wt} \%$ in $t$-butanol, $0.05 \mathrm{mmol})$. The reaction was stirred at 
room temperature for $3 \mathrm{~h}$ and was then diluted with diethyl ether $(20 \mathrm{~mL})$. The organics were extracted with aq. $\mathrm{HCl}$ solution $(20 \mathrm{~mL}, 10 \%)$ followed by sat. $\mathrm{NaHCO}_{3}$ solution (2 x $50 \mathrm{~mL}$ ). The organics were dried over $\mathrm{MgSO}_{4}$ and the drying agent removed by filtration before the mixture was concentrated under reduced pressure. The crude product was purified on a silica column with $4 \%$ methanol in a 1:1 mixture of diethyl ether and dichloromethane as eluent and gave the major diol 15 in $46 \%$ yield (64 mg, $0.19 \mathrm{mmol}$ ) and the minor diol 16 in $25 \%$ yield ( $35 \mathrm{mg}, 0.10 \mathrm{mmol}$ ) after two columns, as well as $2 \%$ of mixed fractions ( $3 \mathrm{mg}, 0.089 \mathrm{mmol}$ ).

Spectroscopic data for 15: ${ }^{1} \mathrm{H} \mathrm{NMR}\left(500 \mathrm{MHz}, \mathrm{CDCl}_{3}\right) \delta 3.96(\mathrm{dd}, 1 \mathrm{H}, J=7.3,12.7 \mathrm{~Hz})$, $3.68(\mathrm{dd}, 1 \mathrm{H}, J=2.0,12.7 \mathrm{~Hz}), 3.43-3.36(\mathrm{~m}, 2 \mathrm{H}), 2.69(\mathrm{ddd}, 1 \mathrm{H}, J=2.0,7.3,7.8 \mathrm{~Hz})$, $2.55(\mathrm{~m}, 1 \mathrm{H}), 2.30(\mathrm{~s}$, broad, $2 \mathrm{H}), 2.0(\mathrm{~d}, 1 \mathrm{H}, J=13.7 \mathrm{~Hz}), 1.82-1.55(\mathrm{~m}, 8 \mathrm{H}), 1.46$ (ddd, $1 \mathrm{H}, J=5.9,12.2,18.3 \mathrm{~Hz}), 1.30(\mathrm{~s}, 3 \mathrm{H}), 1.28(\mathrm{~s}, 3 \mathrm{H}), 1.21(\mathrm{~s}, 3 \mathrm{H}), 1.12(\mathrm{~m}, 1 \mathrm{H})$, 1.00 (ddd, $1 \mathrm{H}, J=4.4,14.2,18.3 \mathrm{~Hz}), 0.90(\mathrm{~s}, 3 \mathrm{H}) .{ }^{13} \mathrm{C} \mathrm{NMR}\left(75 \mathrm{MHz}, \mathrm{CDCl}_{3}\right) \delta 215.0$, $74.9,73.8,70.9,59.9,51.9,48.8,47.0,42.8,40,8,39.5,34.6,34.5,32.6,32.5,26.9,26.7$, 24.8, 24.6, 23.2. IR (neat, $\mathrm{cm}^{-1}$ ) 3380, 2959, 2849, 1687, 1159, 1097, 1064, 1012 HRMS (ESI) calcd. for $\mathrm{C}_{20} \mathrm{H}_{32} \mathrm{O}_{4} \mathrm{Na} 359.2193$ found 359.2189 .

Spectroscopic data for 16: ${ }^{1} \mathrm{H}$ NMR $\left(500 \mathrm{MHz}, \mathrm{CDCl}_{3}\right) \delta 3.99(\mathrm{dd}, 1 \mathrm{H}, J=7.3,13.1 \mathrm{~Hz})$, $3.72(\mathrm{dd}, 1 \mathrm{H}, J=2.1,13.1 \mathrm{~Hz}), 3.48(\mathrm{ddd}, 1 \mathrm{H}, J=4.9,6.1,11.6 \mathrm{~Hz}), 3.11$ (ddd, $1 \mathrm{H}, J=$ 3.4, 11.9, $15.0 \mathrm{~Hz}), 2.66$ (1H, ddd, $J=2.1,7.3,9.2 \mathrm{~Hz}), 2.58(\mathrm{~m}, 1 \mathrm{H}), 1.98(\mathrm{~m}, 1 \mathrm{H}), 1.92$ - $1.87(\mathrm{~m}, 2 \mathrm{H}), 1.86(\mathrm{~s}, 1 \mathrm{H},(\mathrm{OH})), 1.73(\mathrm{~d}, 1 \mathrm{H}, J=6,1 \mathrm{~Hz},(\mathrm{OH})), 1.70-1.62(\mathrm{~m}, 5 \mathrm{H})$, 1.51 (ddd, $1 \mathrm{H}$, broad), 1.44 (ddd, $1 \mathrm{H}$, broad), $1.31\left(1 \mathrm{H}\right.$, hidden by $\left.\mathrm{CH}_{3}\right), 1.31(\mathrm{~s}, 6 \mathrm{H})$, 
$1.20(\mathrm{~s}, 3 \mathrm{H}), 1.01(\mathrm{~m}, 1 \mathrm{H}), 0.90(\mathrm{~s}, 3 \mathrm{H}) .{ }^{13} \mathrm{C} \mathrm{NMR}\left(75 \mathrm{MHz}, \mathrm{CDCl}_{3}\right)$ \& 213.7, 74.0, 73.6, $70.8,60.3,51.3,50.7,44.1,43.3,39.9,39.5,34.3,34.0,33.9,32.6,27.3,27.0,25.2,24.2$, 23.1. IR (neat, $\mathrm{cm}^{-1}$ ) 3457, 3397, 2918, 1687, 1452, 1367, 1303, 1088, 1059. HRMS (ESI) calcd. for $\mathrm{C}_{20} \mathrm{H}_{32} \mathrm{O}_{4} \mathrm{Na} 359.2193$ found 359.2196 .

\section{Synthesis of [1,5,5-Trimethyl-9-0xo-10-(2-oxo-propyl)-6-oxa-}

\section{tricyclo[6.3.2.0 4,13 ]tridec-11-yl]-acetaldehyde (17)}

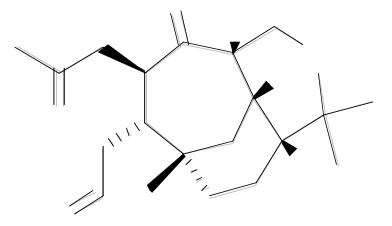

To a stirred solution of the diol $15(46 \mathrm{mg}, 0.13 \mathrm{mmol})$ in methanol $(5.8 \mathrm{~mL})$ at room temperature was added a mixture of sodium periodate $(88 \mathrm{mg}, 0.41 \mathrm{mmol})$ in water $(3.5$ $\mathrm{mL}$ ). The reaction was stirred at room temperature for $12 \mathrm{~h}$ then quenched by adding ether $(25 \mathrm{~mL})$ and extracted with a saturated brine solution $(2 \times 25 \mathrm{~mL})$. The organic layer was dried over magnesium sulfate and concentrated under reduced pressure. The product was purified on a silica column with $20 \%$ acetone in hexane as eluent and gave the aldehyde in $81 \%$ (37 mg, $0.11 \mathrm{mmol})$ yield. ${ }^{1} \mathrm{H}$ NMR $\left(500 \mathrm{MHz}, \mathrm{CDCl}_{3}\right) \delta 9.80(\mathrm{~d}$, $1 \mathrm{H}, J=2.2 \mathrm{~Hz}), 4.16(\mathrm{dd}, 1 \mathrm{H}, J=12.8,5.5 \mathrm{~Hz}), 3.85(\mathrm{dd}, 1 \mathrm{H}, J=12.8,6.6 \mathrm{~Hz}), 3.37$ (ddd, $1 \mathrm{H}, J=16.8,10.3,3.7 \mathrm{~Hz}) 2.97(\mathrm{dd}, 1 \mathrm{H}, J=17.6,10.3 \mathrm{~Hz}), 2.85$ (ddd, $1 \mathrm{H}, J=9.9$, 9.9, $5.9 \mathrm{~Hz}), 2.58(\mathrm{dd}, 1 \mathrm{H}, J=18.3,2.2 \mathrm{~Hz}), 2.54-2.40(\mathrm{~m}, 3 \mathrm{H}), 2.07(\mathrm{~s}, 3 \mathrm{H}), 1.92(\mathrm{~m}$, 1H), $1.86-1.81(\mathrm{~m}, 2 \mathrm{H}), 1.70-1.63(\mathrm{~m}, 2 \mathrm{H}), 1.41(\mathrm{~m}, 1 \mathrm{H}), 1.32-1.26(\mathrm{~m}, 1 \mathrm{H}), 1.28(\mathrm{~s}$, 3H), $1.20(\mathrm{~s}, 3 \mathrm{H}), 1.06(\mathrm{~m}, 1 \mathrm{H}), 0.89(\mathrm{~s}, 3 \mathrm{H}) .{ }^{13} \mathrm{C} \mathrm{NMR}\left(125 \mathrm{MHz}, \mathrm{C}_{6} \mathrm{D}_{6}\right) \delta$ 212.4, 205.9, $200.3,73.0,60.2,52.1,50.1,47.5,46.3,42.9,42.2,41.2,35.2,34.6,33.7,29.5,26.4$, 
26.2, 26.0, 24.2. IR (neat, $\mathrm{cm}^{-1}$ ) 2969, 2919, 2720, 1722, 1713, 1691, 1367, 1215, 1096, 1076 HRMS (ESI) calcd. for $\mathrm{C}_{20} \mathrm{H}_{30} \mathrm{O}_{4} \mathrm{Na} 357.2036$ found 357.2030 .

Synthesis of 5-epi-10-epi-vibsanin E (19) and 3-Methyl-but-2-enoic acid (Z)-2-[1,5,5trimethyl-9-oxo-10-(2-oxo-propyl)-6-oxa-tricyclo[6.3.2.0 ${ }^{4,13}$ ]tridec-11-yl]-vinyl ester (18)
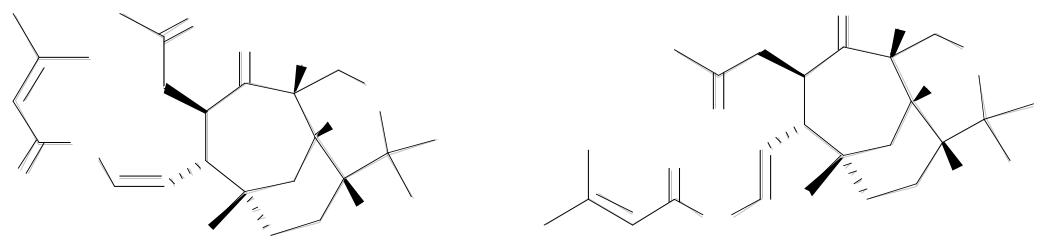

To a stirred solution of the aldehyde $\mathbf{1 7}(13.2 \mathrm{mg}, 0.0395 \mathrm{mmol})$ in pyridine $(1 \mathrm{~mL})$ was added 3,3-dimethylacrylicanhydride $(10.6 \mathrm{mg}, 0.0581 \mathrm{mmol})$ and 4-pyrrolidinopyridine ( $8.5 \mathrm{mg}, 0.0577 \mathrm{mmol}$ ). The mixture was heated to reflux for $3 \mathrm{~h}$ and then allowed to cool to room temperature and ether $(20 \mathrm{~mL})$ was added to the crude mixture. The organic layer was extracted with water $(2 \mathrm{X} 20 \mathrm{~mL})$ followed by a saturated sodium bicarbonate solution $(2 \times 20 \mathrm{~mL})$. The organics were dried over magnesium sulfate, filtered and the solvents were evaporated to yield $22 \mathrm{mg}$ of crude product. This was purified on silica column with $1 \%$ methanol and $3 \%$ acetone in dichloromethane to yield a 2:1 mixture of trans and cis 5-epi-10-epi-vibsanin E (4.8 $\mathrm{mg}, 0,012 \mathrm{mmol}$,) favoring the trans isomer. This mixture was separated on a preparative HPLC system to deliver the trans isomer 19 in $19 \%$ (3.1 mg, $0.075 \mathrm{mmol})$ yield and the cis isomer 18 in $10 \%(1.6 \mathrm{mg}, 0.038 \mathrm{mmol})$ yield. HPLC system: $1 \%$ isopropanol in hexane, $15 \mathrm{~mL} / \mathrm{min}$ at 276 psi. Preparative column: Dynamax 60A, $30 \mathrm{~cm}$ long, $2.5 \mathrm{~cm}$ outer diameter. 
Spectroscopic data for 5-epi-10-epi-vibsanin E (86): ${ }^{1} \mathrm{H}$ NMR $\left(500 \mathrm{MHz}, \mathrm{C}_{6} \mathrm{D}_{6}\right) \delta 7.26$ $(\mathrm{d}, 1 \mathrm{H}, J=12.4 \mathrm{~Hz}), 5.71(\mathrm{qq}, 1 \mathrm{H}, J=1.5 \mathrm{~Hz}), 5.15(\mathrm{dd}, 1 \mathrm{H}, J=12.4,10.9 \mathrm{~Hz}), 4.51$ (dd, 1H, $J=12.7,4.9 \mathrm{~Hz}), 3.95$ (dd, 1H, $J=12,7,7.3 \mathrm{~Hz}), 3.54$ (ddd, 1H, $J=11.8,9.8$, $3.7 \mathrm{~Hz}$ ), 2.91 (ddd, 1H, $J=7.3,6.7,4.4 \mathrm{~Hz}), 2.84$ (dd, 1H, $J=18.0,3.7 \mathrm{~Hz}), 2.37$ (dd, 1H, $J=18.0,9.8 \mathrm{~Hz}), 2.12(\mathrm{dddd}, 1 \mathrm{H}, J=10.1,4.7 \mathrm{~Hz}), 2.07(\mathrm{~d}, 3 \mathrm{H}, J=1.5 \mathrm{~Hz}), 1.71$ (m, 2H) $1.62(\mathrm{~s}, 3 \mathrm{H}) 1.57(\mathrm{dd}, 1 \mathrm{H}, J=11.8,10.9 \mathrm{~Hz}), 1.51(\mathrm{~d}, \mathrm{br}, 1 \mathrm{H}, J=15.0 \mathrm{~Hz}), 1.38$ (d, $3 \mathrm{H}, J=1.5 \mathrm{~Hz}), 1.34(\mathrm{~m}, 1 \mathrm{H}), 1.28(\mathrm{dd}, 1 \mathrm{H}, J=14.6,6.4 \mathrm{~Hz}), 1.14(\mathrm{~s}, 3 \mathrm{H}), 1.06(\mathrm{~s}$, $3 \mathrm{H}), 0.97(\mathrm{ddd}, 1 \mathrm{H}, J=9.4,4.7 \mathrm{~Hz}), 0.72(\mathrm{~s}, 3 \mathrm{H}), 0.69(\mathrm{dd}, 1 \mathrm{H}, J=14.6,4.9 \mathrm{~Hz}) .{ }^{13} \mathrm{C}$ NMR (125 MHz, $\left.\mathrm{C}_{6} \mathrm{D}_{6}\right) \delta$ 212.4, 206.2, 163.3, 160.1, 137.7, 115.0, 114.8, 73.1, 60.4, $53.4,50.5,47.7,47.5,42.4,40.6,34.9,34.3,33.9,29.5,27.1,26.4,26.1,25.8,24.4,20.3$ IR $\left(\right.$ neat, $\left.\mathrm{cm}^{-1}\right)$ 2962, 2925, 1728, 1712, 1687, 1642, 1450, 1356, 1221, 1136, 1091, 943. HRMS (ESI) calcd. for $\mathrm{C}_{25} \mathrm{H}_{36} \mathrm{O}_{5} \mathrm{Na} 439.2455$ found 439.2458

Spectroscopic data for 3-Methyl-but-2-enoic acid (Z)-2-[1,5,5-trimethyl-9-oxo-10-(2oxo-propyl)-6-oxa-tricyclo[6.3.2. $0^{4,13}$ ]tridec-11-yl]-vinyl ester 85: ${ }^{1} \mathrm{H}$ NMR $(500 \mathrm{MHz}$, $\left.\mathrm{C}_{6} \mathrm{D}_{6}\right) \delta 7.44(\mathrm{~d}, 1 \mathrm{H}, J=6.6 \mathrm{~Hz}), 5.66(\mathrm{qq}, 1 \mathrm{H}, J=0.9,0.6 \mathrm{~Hz}), 4.58(\mathrm{dd}, 1 \mathrm{H}, J=12.5$, $5.1 \mathrm{~Hz}), 4.33$ (dd, 1H, $J=11.0,6.6 \mathrm{~Hz}), 3.95$ (dd, 1H, $J=12.5,7.0 \mathrm{~Hz}), 3.43$ (ddd, $1 \mathrm{H}, J$ $=12.5,9.8,3.7 \mathrm{~Hz}), 3.01(\mathrm{dd}, 1 \mathrm{H}, J=18.3,9.8 \mathrm{~Hz}), 2.96(\mathrm{ddd}, 1 \mathrm{H}, J=12.2,7.0,5.1 \mathrm{~Hz})$, $2.58(\mathrm{dd}, 1 \mathrm{H}, J=12.5,11.0 \mathrm{~Hz}), 2.45(\mathrm{dd}, 1 \mathrm{H}, J=18.3,3.7 \mathrm{~Hz}), 2.14(\mathrm{~m}, 1 \mathrm{H}), 2.05(\mathrm{~d}$, $3 \mathrm{H}, J=0.6 \mathrm{~Hz}), 1.79-1.71(\mathrm{~m}, 1 \mathrm{H}), 1.68$ (broad dd, 1H, $J=14.6 \mathrm{~Hz}), 1.62(\mathrm{~s}, 3 \mathrm{H}), 1.40$ (m, 1H), $1.36(\mathrm{~d}, 3 \mathrm{H}, J=0.9 \mathrm{~Hz}), 1.33(\mathrm{dd}, 1 \mathrm{H}, J=14.6,6.4 \mathrm{~Hz}), 1.17(\mathrm{~s}, 3 \mathrm{H}), 1.08$ (s, $3 \mathrm{H}), 1.06(\mathrm{dd}, 1 \mathrm{H}, J=6.4 \mathrm{~Hz}), 1.00(\mathrm{~m}, 1 \mathrm{H}), 0.89(\mathrm{~s}, 3 \mathrm{H}), 0.75(\mathrm{~m}, 1 \mathrm{H}) .{ }^{13} \mathrm{C}$ NMR $(125$ $\left.\mathrm{MHz}, \mathrm{C}_{6} \mathrm{D}_{6}\right) \delta 212.8,206.3,163.1,160.4,135.8,115.0,114.0,73.1,60.5,53.5,47.5$, 
$47.14,47.05,42.9,40.8,35.5,34.1,33.8,29.4,27.0,26.42,26.37,26.1,24.5,20.4$. IR

(neat, $\mathrm{cm}^{-1}$ ) 2970, 2905, 1728, 1687, 1642, 1221, 1131. HRMS (ESI) calcd. for $\mathrm{C}_{25} \mathrm{H}_{36} \mathrm{O}_{5} \mathrm{Na} 439.2455$ found 439.2457

\section{Section C: Relative Stereochemistry for 5-epi-10-epi-Vibsanin E (19)}

Compounds 13 - 17 did not give characteristic NOE correlations in order to establish the relative stereochemistry of the two alkenes $\mathbf{1 3}$ and $\mathbf{1 4}$. Furthermore, none of these compounds were suited for X-ray crystallography either. Because of this the products were taken through to the next steps in the synthesis. In order to establish the correct relative stereochemistry for the synthesized compound $\mathbf{1 8}$ and 19 nOe studies were undertaken and this data was compatible with a trans addition for the photochemical addition of isoprene across the enone 7 , but epimeric at the 5 and 10 position compared to the natural product. This is clearly seen by inspecting the nOe correlations for $\mathbf{1 9}$. The proton at position 5 is quite characteristic for vibsanin $\mathrm{E}$ type of compounds as it is a ddd typically in the $3.0-3.6 \mathrm{ppm}$ range with $\mathrm{C}_{6} \mathrm{D}_{6}$ as the NMR solvent. For the synthesized compound this proton interacts with position 12,18 and the dd signal from the proton on the enol ether. It also has to be "down" because of the interaction with the proton at position 18 , since the geminal proton at this position interacts with the hydrogen at position 2, which is known to be "up" due to the convex shape of these diterpenes. A very powerful indication for the suggested structure is the correlation between position 5 and 12 . The relevant proton at position 12 is found at $1.70 \mathrm{ppm}$ because it is shielded by the carbonyl group just above it in space. This correlation can only be observed if the 
proton at position 5 is "down". Since this hydrogen also shows an nOe from the proton on enol ether, the proton at position 10 has to be "up", Figure 14.

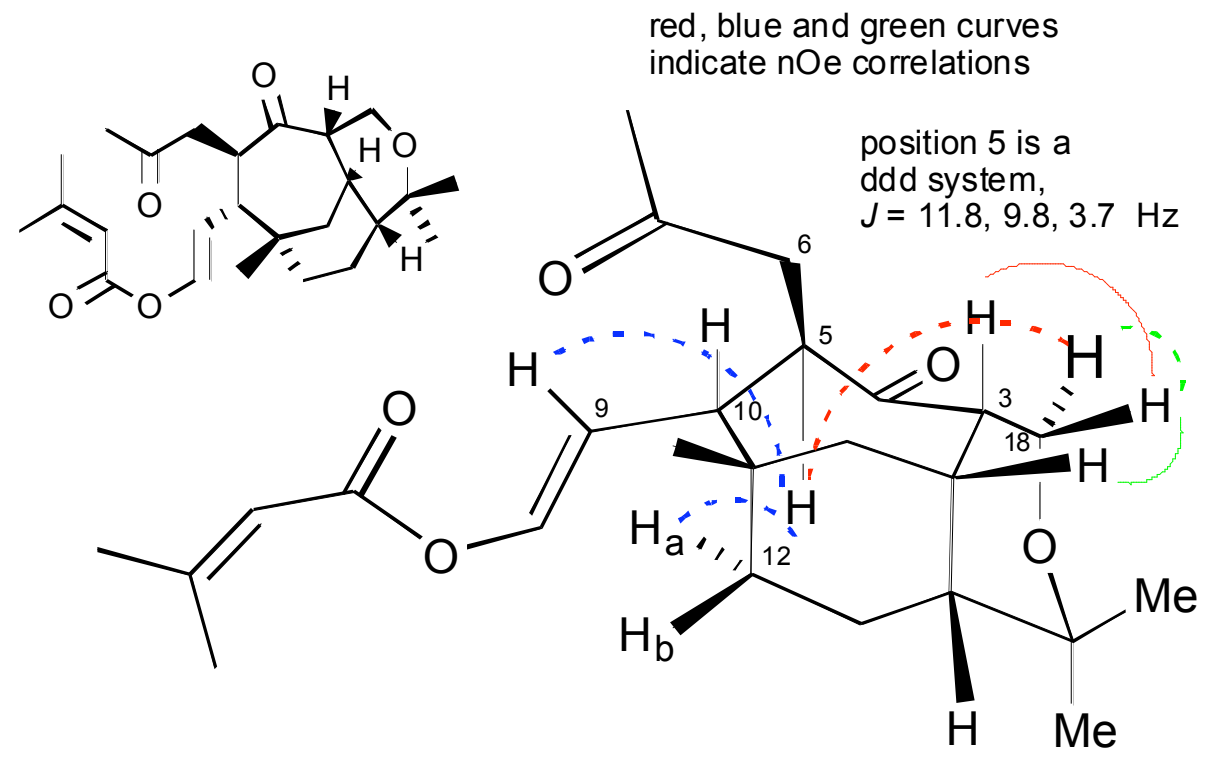

Figure 1. nOe Correlations for 5-epi-10-epi-Vibsanin E 19

\section{Section D: ${ }^{1} \mathrm{H}$ NMR Spectra}

